\title{
NOVEL RHEOLOGICAL MODEL FOR THE INELASTIC BLOOD RHEOLOGY: ITS DERIVATION AND VALIDATION
}

\author{
Ternik, P. \& Marn, J. \\ University of Maribor, Faculty of Mechanical Engineering, \\ Smetanova ulica 17, 2000 Maribor, Slovenia \\ E-Mail: primoz.ternik@uni-mb.si; jure.marn@uni-mb.si
}

\begin{abstract}
A novel approach to rheological modelling of human blood is proposed. The fundamental equation relating the apparent wall shear rate to the wall shear stress is used to derive the general relationship between the shear stress and the shear rate. Then the Parabolic model is tested against available experimental data for blood and compared to the well known and widely used Power law. Numerical analysis of non-Newtonian (blood) as well as Newtonian fluid flow in common carotid geometry is used to compare numerical results with theoretical expressions for validation of Parabolic model.

(Received in May 2007, accepted in September 2007. This paper was with the authors 1 month for 1 revision.)
\end{abstract}

Key Words: Blood Rheology, Shear-Thinning Fluid, Parabolic Model, Numerical Modelling

\section{INTRODUCTION}

The complexity of the human cardiovascular system features a tremendous variety of districts like large arteries, veins of medium calibres as well as capillaries. Their size ranges from few centimetres in diameter down to few micrometers. Except for the very thin and tiny capillaries, the human blood flow can be treated as an incompressible continuum [1]. As such, its hemodynamic behaviour can be described by its velocity and pressure fields, related by the momentum and mass conservation equations.

Whole blood consists of formed elements that are suspended in an aqueous saline solution called plasma. The majority of formed elements are red blood cells (RBC) and they occupy approximately $45 \%$ of blood volume and therefore dominate the rheological blood behaviour [2]. At low shear rates (values less than $100 \mathrm{~s}^{-1}$ [3] or even $10 \mathrm{~s}^{-1}$ [2]) the RBC aggregate face to face and form a rouleaux which increases the viscosity of blood. But on the other hand, these rouleaux segregate and RBC align with the flow at high shear rates $\left(>200 \mathrm{~s}^{-1}\right)$ and at even higher shear rates RBC start to deform and decrease the viscosity of blood.

Various researchers have proposed a variety of constitutive equations (rheological models) representing the inelastic viscous behaviour of blood. Such rheological models form the solid and very useful basis of blood representation in a computational fluid dynamics (CFD). CFD software enables the numerical simulation of blood flow in a number of complex structures; e.g. carotid artery bifurcation [4, 5, 6], arteries [7, 8, 9], aortic arch [10]. In most of numerical studies the blood flow was modelled as an incompressible Newtonian fluid where the non-Newtonian rheology of blood was ignored [4, 10,12]. But on the other hand several numerical studies indicate that the influence of shear-thinning properties of blood is significant for the blood flow not only in small arteries but also in large arteries. Only a few studies incorporated the shear-thinning non-Newtonian behaviour of blood. Most widely used rheological models in those studies was Carreau-Yasuda model [5, 6, 7], while some other studies incorporated Power Law model [7] and Herschel-Bulkley model [13]. The most important outcome of studies where non-Newtonian blood properties were accounted for is 
the different wall shear stress distribution at low shear rates, which is an important hemodynamic condition attributed to formation of atherosclerotic plaques. It is also known that the non-Newtonian fluid behaviour results in different flow dynamics in comparison to Newtonian fluid not only in 2-dimensional [14] but also in 3-dimensional geometries [15].

Although many rheological models for blood already exist in literature and are used for numerical modelling purposes we have recently shown that there is still a place for a new rheological model [16]. A Quadratic model was proven to do much better job in correlating the experimental data (shear stress vs. shear rate and wall shear stress vs. apparent wall shear rate) not only for an electrostatic ash and water but also for a corn-starch and water as well as glass-beads and water mixture flows. Its applicability was brought into a day light for both two- and three-dimensional shear-thickening fluid flows $[14,15]$ proving the usefulness of a better suited rheologial model in comparison to a well known and widely used Power law model.

It is therefore the aim of present study to develop a new rheological model that will account for inelastic and shear-thinning viscous behaviour of blood. In Section 2, the Parabolic model is presented along with equations for the velocity profile, average velocity and generalized Reynolds number. Section 3 deals with the determination of Parabolic model parameters for human blood. Numerical modelling of blood flow in common carotid geometry is presented in Section 4, while numerical results are presented in Section 5.

\section{PARABOLIC MODEL}

The fundamental equation of the model, giving the relationship between the apparent wall shear rate $(8 \bar{v} / D)$ and the wall shear stress $\tau_{W}$ for the fully developed pipe flow is:

$$
\left(\frac{8 \bar{v}}{D}\right)=A \tau_{W}^{2}+B \tau_{W}
$$

from which the wall shear stress is expressed as:

$$
\tau_{W}=\frac{-B+\sqrt{B^{2}+4 A(8 \bar{v} / D)}}{2 A}
$$

omitting the second (negative) solution of Equation (1).

Differentiation of Equation (1) with respect to $(8 \bar{v} / D)$ gives:

$$
\frac{d(8 \bar{v} / D)}{d \tau_{W}}=2 A \tau_{W}+B
$$

General relationship for all generalized Newtonian fluids pipe flow [17]:

$$
\dot{\gamma}_{W}=\frac{3}{4}\left(\frac{8 \bar{v}}{D}\right)+\frac{\tau_{W}}{4}\left[\frac{d(8 \bar{v} / D)}{d \tau_{W}}\right]
$$

and Equation (3) are combined to arrive at:

$$
\dot{\gamma}_{W}=\frac{5 A}{4} \tau_{W}^{2}+B \tau_{W}
$$

and finally: 


$$
\tau_{W}=\frac{-2 B+2 \sqrt{B^{2}+5 A \dot{\gamma}_{W}}}{5 A}
$$

One can see that the Equation (5) is a parabolic equation for the wall shear stress from which the general relationship between the shear stress and shear rate can be derived as:

$$
\tau_{i j}=\frac{-2 B+2 \sqrt{B^{2}+5 A|\dot{\gamma}|}}{5 A|\dot{\gamma}|} \dot{\gamma}_{i j}
$$

where $|\dot{\gamma}|=\sqrt{\frac{1}{2} \sum_{i} \sum_{j} \dot{\gamma}_{i j} \dot{\gamma}_{j i}}$ is a II. invariant of symmetrical rate of deformation tensor with Cartesian coordinates [18] $\dot{\gamma}_{i j}=\left(\frac{\partial v_{i}}{\partial x_{j}}+\frac{\partial v_{j}}{\partial x_{i}}\right)$.

\subsection{Velocity profile}

For the validation of the Parabolic model it is beneficial to find out if an analytical solution to the velocity profile for a fully developed straight pipe flow may be constructed.

For the straight pipe flow the following relationships are used:

$$
\dot{\gamma}_{r}=-\left(\frac{d v_{x}}{d r}\right), \quad \tau_{r}=\frac{\Delta p}{2 L} r
$$

and combined with Equation (5) to arrive at:

$$
\frac{d v_{x}}{d r}=-\frac{5 A}{4} \frac{\Delta p^{2}}{4 L^{2}} r^{2}-B \frac{\Delta p}{2 L} r
$$

and:

$$
\int d v_{x}=-\int \frac{5 A}{4} \frac{\Delta p^{2}}{4 L^{2}} r^{2} d r-\int B \frac{\Delta p}{2 L} r d r
$$

Equation (8) is integrated with an appropriate ("no-slip" at solid walls) boundary condition:

$$
r=R \rightarrow v_{x}(r)=0
$$

to arrive at the following equation for the velocity profile:

$$
v_{x}(r)=\frac{5 A}{48}\left(\frac{\Delta p}{L}\right)^{2}\left(R^{3}-r^{3}\right)+\frac{B}{4}\left(\frac{\Delta p}{L}\right)\left(R^{2}-r^{2}\right)
$$

The maximum velocity occurs at the pipe centre $(r=0)$ :

$$
v_{x, \max }=\frac{5 A}{48}\left(\frac{\Delta p}{L}\right)^{2} R^{3}+\frac{B}{4}\left(\frac{\Delta p}{L}\right) R^{2}
$$

The average velocity: 


$$
\bar{v}=\frac{\int_{0}^{R} v_{x}(r) 2 \pi r d r}{\int_{0}^{R} 2 \pi r d r}
$$

is derived using the Equation (9) as:

$$
\bar{v}=\frac{A}{16}\left(\frac{\Delta p}{L}\right)^{2} R^{3}+\frac{B}{8}\left(\frac{\Delta p}{L}\right) R^{2}
$$

\subsection{Generalized Reynolds number}

It is beneficial for comparison between various rheological models if an appropriate dimensionless parameter such as generalized Reynolds number is constructed. The frictional pressure loss for fully developed and laminar flow is included in the dimensionless Fanning friction factor:

$$
f=\frac{\frac{\Delta p}{4 L} D}{\frac{1}{2} \rho \bar{v}^{2}}=\frac{\tau_{W}}{\frac{1}{2} \rho \bar{v}^{2}}
$$

Since the Fanning friction factor equals $f=16 /$ Re for the straight pipe flow [17], one can derive the following expression for Parabolic model generalized Reynolds number:

$$
\operatorname{Re}=\frac{16 A \rho \bar{v}^{2}}{-B+\sqrt{B^{2}+4 A\left(\frac{8 \bar{v}}{D}\right)}}
$$

\section{PARABOLIC MODEL PARAMETERS DETERMINATION}

Using the experimental data for blood [19] the parameters $A$ and $B$ of the proposed model were determined with the non-linear least squares regression analysis [20]. Similar procedure was used to determine the parameters of Quadratic model for shear-thickening fluids [16].

\subsection{Non-linear least squares regression analysis}

Relationship between the non-linear equation and the experimental data is expressed as:

$$
\tau_{i}=f\left(\dot{\gamma}_{i} ; A, B\right)+e_{i}
$$

where $\tau_{i}$ is a measured value of the dependent variable, $f\left(\dot{\gamma}_{i} ; A, B\right)$ is the function of the independent variable $\dot{\gamma}_{i}$ and a non-linear function of parameters $A$ and $B$, and $e_{i}$ is a random error. The non-linear model is expanded in a Taylor series around the parameter values and higher order terms are dropped. Now Equation (14) reads in a matrix form:

$$
\{D\}=\left[Z_{j}\right]\{\Delta P\}+\{\Delta E\}
$$


where $\left[Z_{j}\right\rfloor$ is the matrix of partial derivatives of the function evaluated at the initial guess $j$, vector $\{D\}$ contains the difference between measurements and function value, and the vector $\{\Delta P\}$ contains changes in parameter values. Applying the least-squares theory to Equation (15) results in:

$$
\left[\left[Z_{j}\right]^{T}\left[Z_{j}\right]\right]\{\Delta P\}=\left\{\left[Z_{j}\right]^{T}\{D\}\right\}
$$

which is solved for $\{\Delta P\}$ and employed to compute the improved values for parameters. This procedure is repeated until:

$$
\left|\varepsilon_{A}\right|=\left|\frac{A_{j+1}-A_{j}}{A_{j+1}}\right| \cdot 100 \%
$$

and:

$$
\left|\varepsilon_{B}\right|=\left|\frac{B_{j+1}-B_{j}}{B_{j+1}}\right| \cdot 100 \%
$$

diminishes bellow the success criterion; i.e.: $\left|\varepsilon_{A}\right|,\left|\varepsilon_{B}\right| \leq 10^{-6}$.

\subsection{Parabolic model for blood}

Experiments with the shear-rheometer in a Couette geometry [19] were used to accommodate the Parabolic model to experimental data for the shear rate $\dot{\gamma}$ and the shear stress $\tau$. Six rheological measurements of the human blood with $47 \%$ hematocrite were analysed and results for the Parabolic model are compared with the widely used Power law model. For the latter parameters $K$ and $n$ were determined using the least-squares method [16].

From Table I as well as Fig. 1 it is clearly evident that the Parabolic model does significantly better job correlating the experimental data for all blood samples. Here $r^{2}$ is so called coefficient of determination (statistics that is widely used to determine how well a regression fits).

Table I: Comparison of Parabolic model and Power law.

\begin{tabular}{c|ccc|ccc} 
& \multicolumn{3}{|c|}{ Parabolic model } & \multicolumn{3}{c}{ Power law } \\
Blood sample & $A\left[1 / \mathrm{Pa}^{2} s\right]$ & $B[1 / \mathrm{Pas}]$ & $r^{2}$ & $K\left[\mathrm{Pas}^{n}\right]$ & $n$ & $r^{2}$ \\
\hline Sample 1 & $1,7180 \mathrm{E}+01$ & $1,9124 \mathrm{E}+02$ & 0,9976 & $1,6482 \mathrm{E}-02$ & $7,4788 \mathrm{E}-01$ & 0,9637 \\
Sample 2 & $1,4360 \mathrm{E}+01$ & $2,0665 \mathrm{E}+02$ & 0,9977 & $1,5684 \mathrm{E}-02$ & $7,4865 \mathrm{E}-01$ & 0,9604 \\
Sample 3 & $1,7730 \mathrm{E}+01$ & $1,9781 \mathrm{E}+02$ & 0,9969 & $1,8050 \mathrm{E}-02$ & $7,2570 \mathrm{E}-01$ & 0,9588 \\
Sample 4 & $1,6186 \mathrm{E}+01$ & $2,0311 \mathrm{E}+02$ & 0,9972 & $1,6751 \mathrm{E}-02$ & $7,3783 \mathrm{E}-01$ & 0,9609 \\
Sample 5 & $1,6595 \mathrm{E}+01$ & $2,0030 \mathrm{E}+02$ & 0,9970 & $1,8569 \mathrm{E}-02$ & $7,1800 \mathrm{E}-01$ & 0,9519 \\
Sample 6 & $1,5093 \mathrm{E}+01$ & $2,0183 \mathrm{E}+02$ & 0,9972 & $1,8478 \mathrm{E}-02$ & $7,1821 \mathrm{E}-01$ & 0,9480
\end{tabular}




\section{NUMERICAL MODELLING}

\subsection{Governing equations}

Let us consider steady, laminar and isothermal flow of incompressible non-Newtonian fluid. For such a flow, the movement is governed by the mass and momentum conservation equations:

$$
\begin{gathered}
\frac{\partial v_{j}}{\partial x_{j}}=0 \\
\rho v_{j} \frac{\partial v_{i}}{\partial x_{j}}=-\frac{\partial p}{\partial x_{i}}+\frac{\partial \tau_{i j}}{\partial x_{j}}
\end{gathered}
$$

In a momentum equation, a constitutive model is needed for the shear stress $\tau_{i j}$. As mentioned in the introduction, it is our intention to use the proposed Parabolic model for blood flow modelling. In all numerical examples presented herein we have used the constants of Parabolic model for blood sample $2\left(A=1.4360 \times 10^{1}\right.$ and $\left.B=2.0665 \times 10^{2}\right)$.

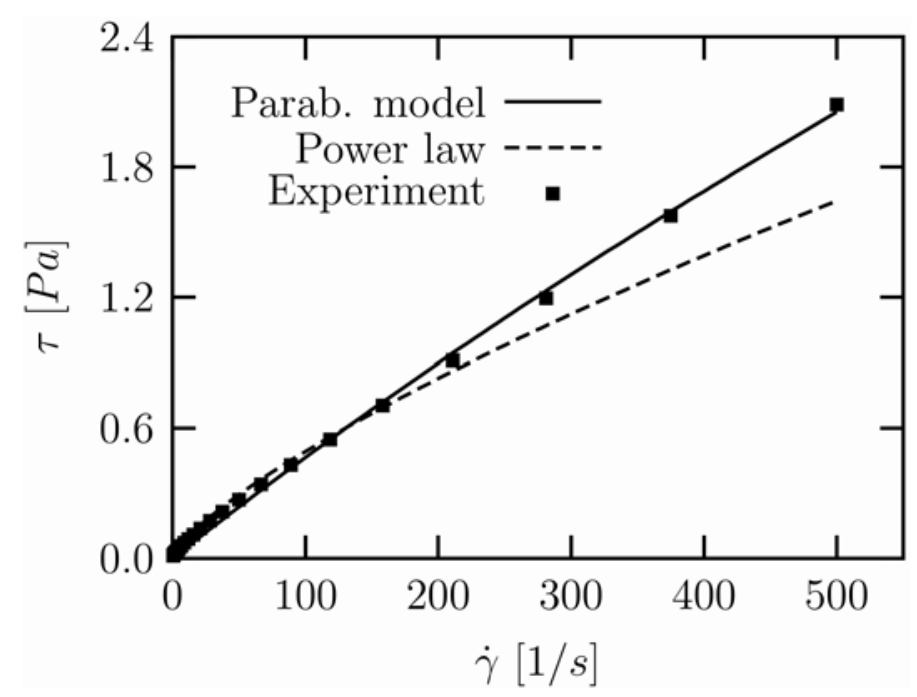

Figure 1: Comparison of experimental data and rheological models.

\subsection{Geometry and boundary conditions}

Geometry of a common carotid as used in the present study is shown on Fig. 2. At the inlet the Neumann boundary conditions (with mass flow restriction) were imposed on a velocity, approximating the fully developed flow conditions, while the outflow boundary was used to impose Dirichlet boundary conditions for pressure $\left(p_{2}=0\right)$ and Neumann boundary conditions (i.e. zero normal gradients) on a velocity.

The common carotid was studied as a rigid pipe with no-slip boundary conditions for the velocity an all walls. 


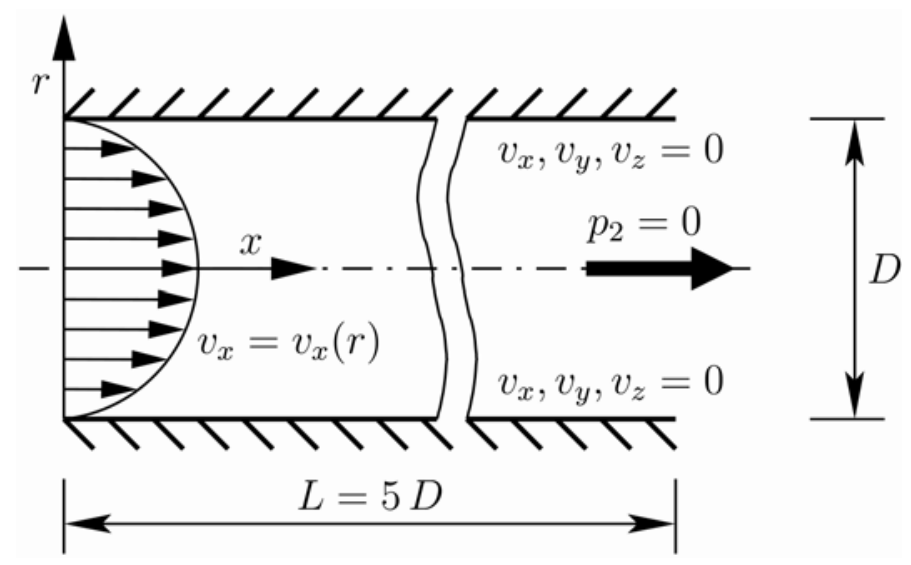

Figure 2: Geometry and boundary conditions; $D=0.0062 \mathrm{~m}$.

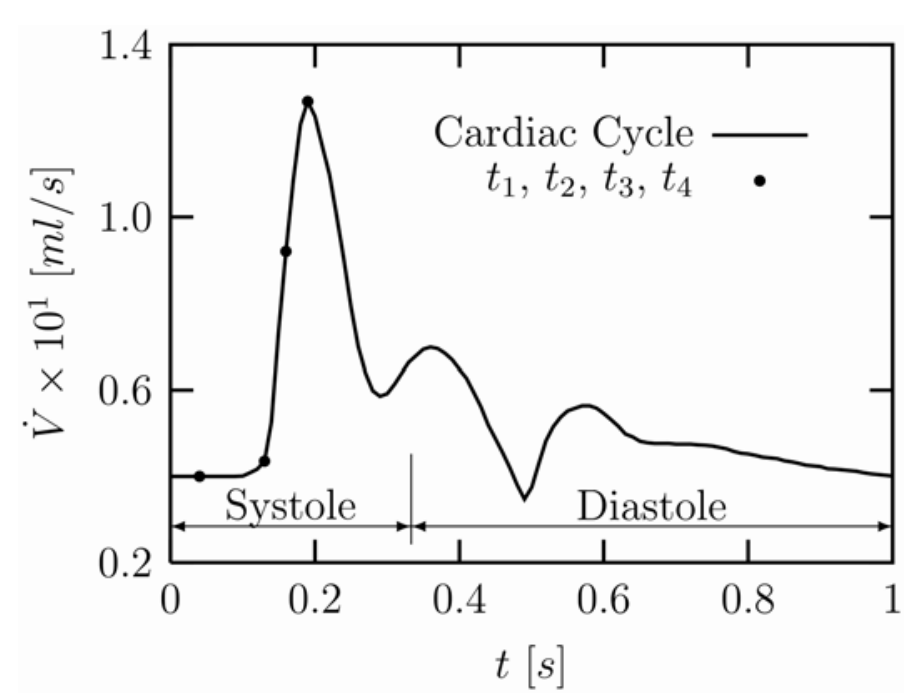

Figure 3: Cardiac cycle.

All numerical examples were performed at four time steps in the systolic phase of a cardiac cycle, Fig. 3. For particular volume flow rate the mass flow and resulting average velocity were determined on basis of blood density and diameter of common carotid, Table II.

Table II: Average velocity and generalized Reynolds number for blood flow.

\begin{tabular}{c|c|c}
$t[s]$ & $\bar{v}[\mathrm{~m} / \mathrm{s}]$ & $\operatorname{Re}$ \\
\hline$t_{1}=0.04$ & $1.3236 E-01$ & 189.1878 \\
$t_{2}=0.13$ & $1.4408 E-01$ & 206.8316 \\
$t_{3}=0.16$ & $3.0468 E-01$ & 461.8246 \\
$t_{4}=0.19$ & $4.1991 E-01$ & 658.7960
\end{tabular}

\subsection{Numerical procedure}

The governing equations were solved using CFX-4.4 computer code which employs the standard finite volume method with all variables defined at the centre of the control volumes populating the physical domain under consideration. Each equation is integrated over each control volume to obtain a discrete equation which connects the variable at the centre of the volume with its neighbours. 
For a spatial discretization the Quadratic upwind differencing scheme [21] was used. It is an upwind scheme (using two upstream points and one downstream point) which is a thirdorder accurate for the advection terms, while all other (diffusion) terms remain second-order.

The mass and momentum equations in three dimensions provide four unknowns: $v_{x}, v_{y}$, $v_{z}$ and $p$. In the solution process, an equation describing the update of the pressure is required but is not explicitly available via the mass or momentum balances. Updated velocity and pressure fields satisfying exactly mass balance and approximately satisfying the discrete momentum equations were obtained by the SIMPLEC algorithm [22], which resolves the coupling between the velocity and pressure.

\section{RESULTS AND DISCUSSION}

\subsection{Nodalization analysis}

First, the influence of a computational mesh refinement on computational results was studied. For this, the blood flow at value of Reynolds number $\mathrm{Re}=658.7960$ was considered and the physical domain of common carotid was populated with three different computational meshes as shown in Fig. 4.
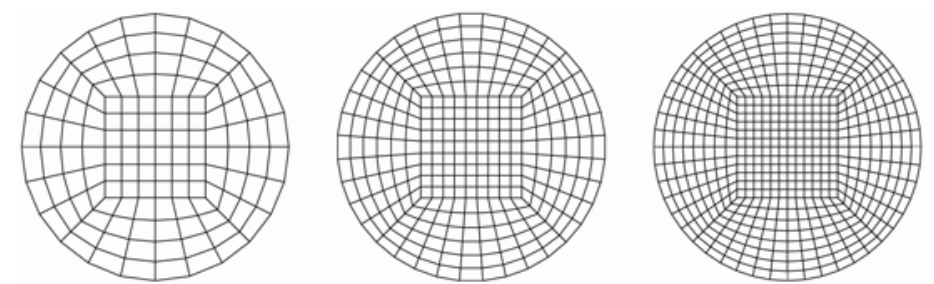

Figure 4: Cross section area of computational domain.

Based on the results for normalized velocity profile, Fig. 5, one can conclude that further computational mesh refinement would not significantly improve the accuracy of numerical procedure. In addition, the authors believe that Mesh III was of sufficient accuracy to model a blood flow in common carotid.

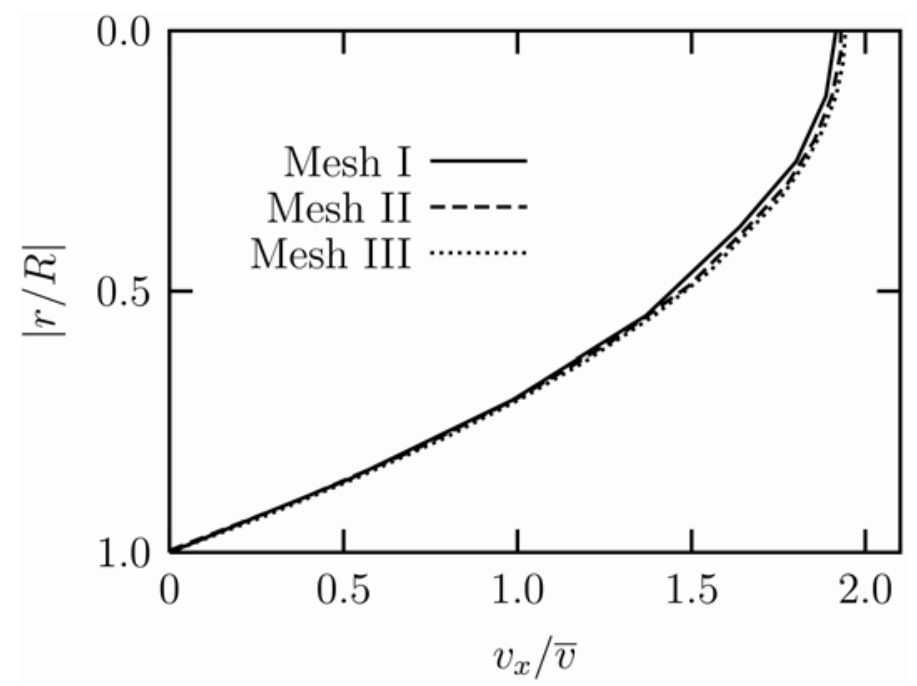

Figure 5: Nodalization analysis results: normalized velocity profile. 


\subsection{Validation of Parabolic model}

Comparison of theoretically and numerically obtained results for blood flow described with the Parabolic model is shown in Fig. 6 and Fig. 7. One can see that numerical results are in excellent agreement with theoretical ones not only for the velocity profile, Fig. 6 (left) but also for the shear stress distribution, Fig. 6 (right), and viscous function, Fig. 7 (left).
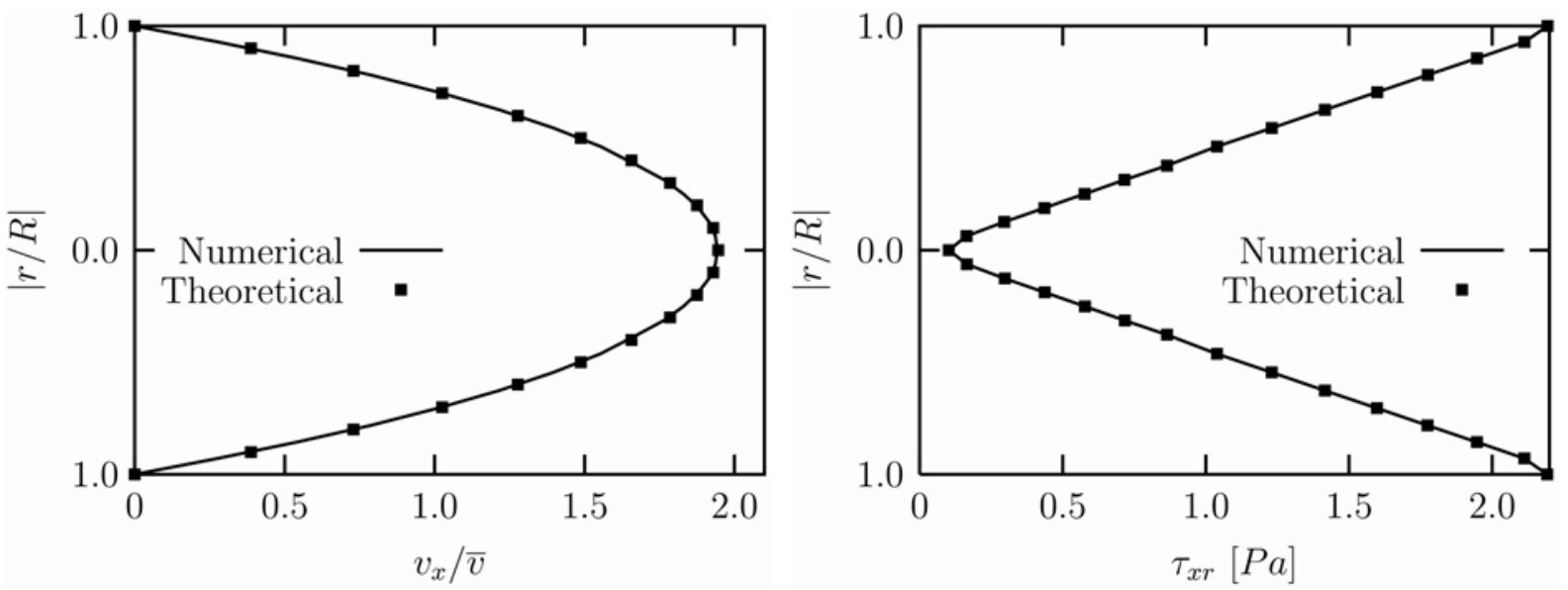

Figure 6: Normalized velocity profile (left) and shear stress distribution (right); $T=0.19 \mathrm{~s}$.
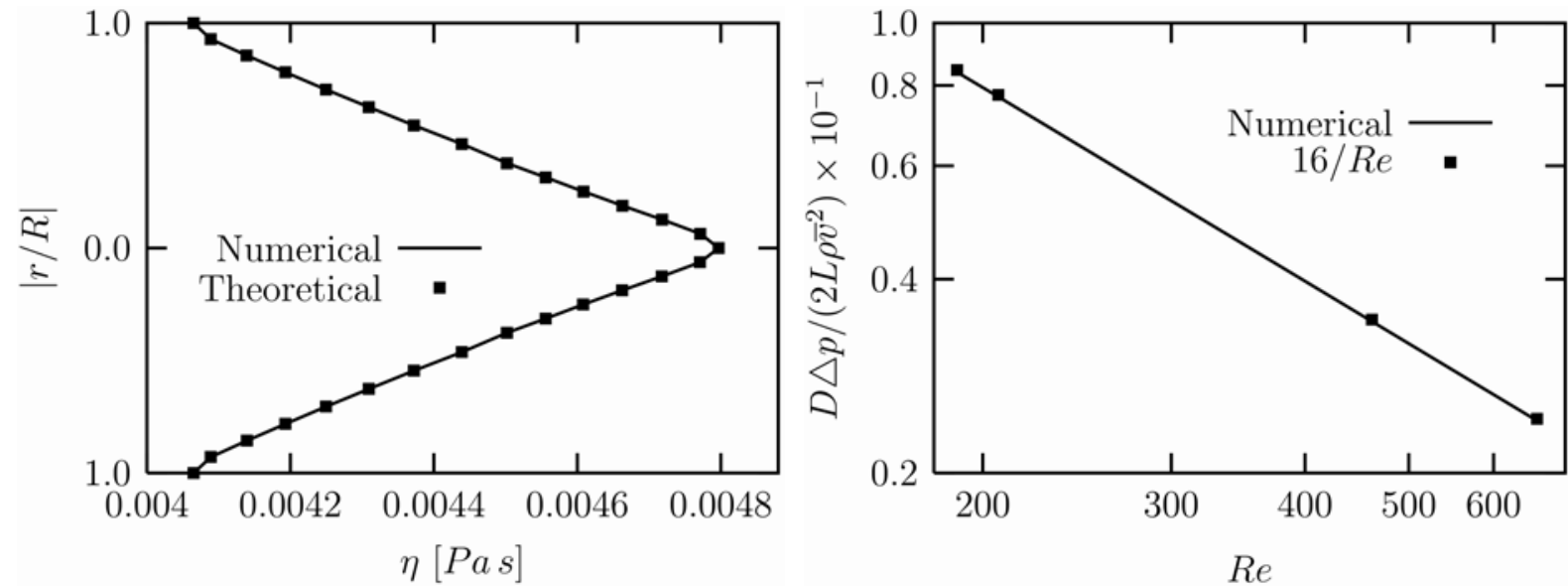

Figure 7: Viscous function (left) for $T=0.19 \mathrm{~s}$, normalized pressure drop (right).

Last but not least, the results for normalized pressure drop, Fig. 7 (right), show very good agreement with theoretical expression and it is therefore our belief that the expression for generalized Reynolds number of a Parabolic model is correctly derived.

\subsection{Parabolic model for Newtonian fluid flow}

It was also our intention to explore the capability of a Parabolic model to describe the limiting (Newtonian) fluid behaviour. For that we have used the following value of parameters $A=1.0000 \times 10^{-8}$ and $B=2.0665 \times 10^{2}$ which result in a constant value of dynamic viscosity $\eta=\frac{1}{B}=4.8391 \times 10^{-3}$ Pas .

As for the non-Newtonian fluid (blood) flow one can see an excellent agreement between numerical and theoretical results for the Newtonian fluid described with the Parabolic model, Fig. 8 and Fig. 9. 

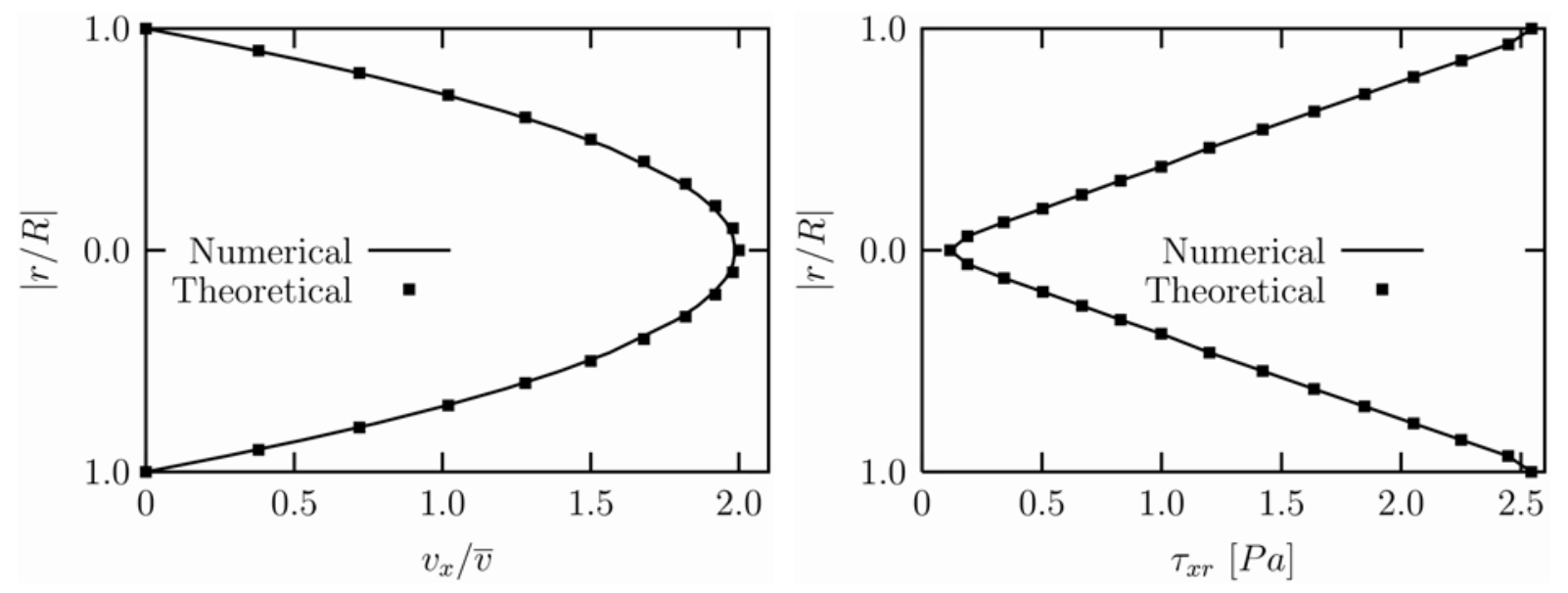

Figure 8: Normalized velocity profile (left) and shear stress distribution (right); $T=0.19 \mathrm{~s}$.

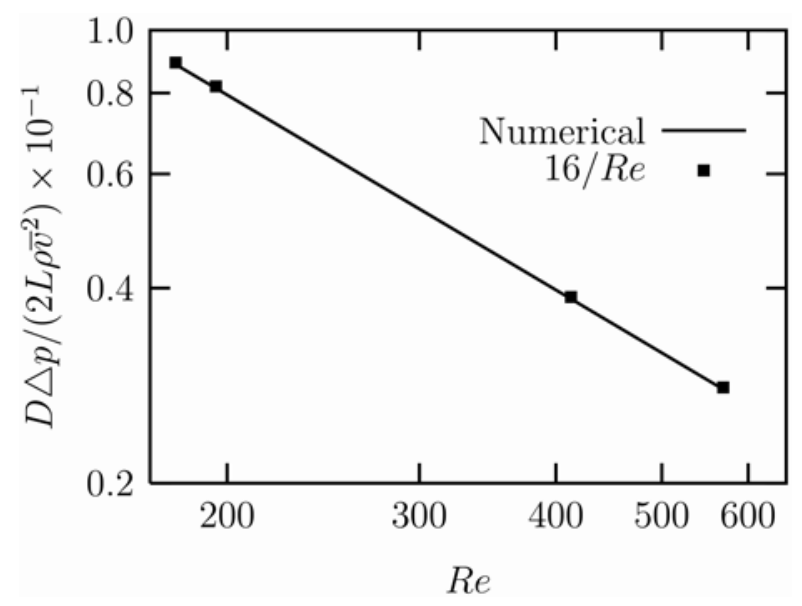

Figure 9: Normalized pressure drop.

Numerical results for Newtonian fluid flow as presented herein are not surprising. The most important outcome of this analysis lies in the fact that the Parabolic model is capable of capturing the Newtonian fluid behaviour and can be therefore used to validate the numerical procedure in more complex geometries where no analytical expressions (for velocity profile) exist and we have to compare our numerical results with results (numerical as well as experimental) of other authors; e.g. Newtonian fluid flow in a pipe bend [15], Newtonian fluid flow in a planar expansions [14] or contractions.

\section{CONCLUSIONS}

The novel rheological model, Parabolic model namely, is proposed to describe purely viscous shear-thinning behaviour of a human blood. Equations for shear stress, velocity profile, average velocity and generalized Reynolds number were derived using the fundamental relationship between the wall shear stress and the apparent wall shear rate of a model.

Results for the shear stress and the shear rate of an actual experimental set up with the shear-rheometer in Couette geometry were used to determine the parameters for Parabolic model as well as for Power law. Comparison of determination coefficients of both rheological models, describing the same shear-thinning behaviour of blood favours the Parabolic model over widely used Power law. 
Using the CFX numerical code the fully developed laminar flow of blood in a common carotid was modelled. Nodalization analysis, using three computational meshes, was performed to study the influence of grid refinement on accuracy of numerical results. For four different time steps (mass flow rates) in a systolic phase of cardiac cycle we have shown an excellent agreement between numerical and theoretical results for both, non-Newtonian (blood) as well as Newtonian fluid, and therefore validated the proposed rheological model.

\section{ACKNOWLEDGEMENTS}

The authors wish to express sincere appreciation to the World Federation of Scientists and the Slovenian Science Foundation for the financial support of the present work within the framework of research entitled "Influence of non-Newtonian rheological models on blood hemodynamic in vascular system” (World Federation of Scientists National Scholarship, Contract Number: SZF - P.Ternik/WFS - 01/2007).

\section{REFERENCES}

[1] Quarteroni, A.; Tuveri, M.; Veneziani, A. (2000). Computational vascular fluid dynamics: problems, models and methods, Computing and Visualization in Science, Vol. 2, 163-197

[2] O’Callaghan, S.; Walsh, M.; McGloughlin, T. (2006). Numerical modelling and non-Newtonian representation of blood in a distal end-to-side vascular bypass graft anastomosis, Medical Engineering \& Physics, Vol. 28, 70-74

[3] Shibeshi, S. S.; Collins, W. E. (2005). The rheology of blood flow in a branched arterial system, Applied Rheology, Vol. 15, 398-405

[4] Botnar, R.; Rappitsch, G.; Scheidegger, M. B.; Liepsch, D.; Perktold, K.; Boesiger, P. (2000). Hemodynamics in the carotid artery bifurcation: a comparison between numerical simulations and in vitro MRI measurements, Journal of Biomechanics, Vol. 33, 137-144

[5] Chen, J.; Lu, X. Y. (2004). Numerical investigation of the non-Newtonian blood flow in a bifurcation model with a non-planar branch, Journal of Biomechanics, Vol. 37, 1899-1911

[6] Gijsen, F. J. H.; van de Vosse, F. N.; Janssen, J. D. (1999).The influence of the non-Newtonian properties of blood in the flow in large arteries: steady flow in a carotid bifurcation model, Journal of Biomechanics, Vol. 32, 601-608

[7] Johnston, B. M.; Johnston, P. R.; Corney, S.; Kilpatrick, D. (2004). Non-Newtonian blood flow in human right coronary arteries: steady state simulations, Journal of Biomechanics, Vol. 37, 709-720

[8] Jung, J.; Lyczkowski, R. W.; Panchal, C. B.; Hassanein, A. (2006). Multiphase hemodynamic simulation of pulsatile flow in a coronary artery, Journal of Biomechanics, Vol. 39, 2064-2073

[9] Chen, J.; Lu, X. Y.; Wang, W. (2006). Non-Newtonian effects of blood flow on hemodynamics in distal vascular graft anastomoses, Journal of Biomechanics, Vol. 39, 1983-1995

[10] Shahcheraghi, N.; Dwyer, H. A.; Cheer, A. Y.; Barakat, A. I.; Rutaganira, T. (2002). Unsteady and Three-Dimensional Simulation of Blood Flow in the Human Aortic Arch, Journal of Biomechanical Engineering (Transactions of the ASME), Vol. 124, 378-387

[11] Shibeshi, S. S.; Collins, W. E. (2005). The rheology of blood flow in a branched arterial system, Applied Rheology, Vol. 15, Issue 6, 398-405

[12] Politis, A. K.; Stavropoulos, G. P.; Christolis, M. N.; Panagopoulos, F. G.; Vlachos, N. S.; Markatos, N. C. (2007). Numerical modelling of simulated blood flow in idealized composite arterial coronary grafts: Steady state simulations, Journal of Biomechanics, Vol. 40, 1125-1136

[13] Cheng, T.; Deville, M. (1996). Pulsatile flow of non-Newtonian fluids through arterial stenoses, Journal of Biomechanics, Vol. 29, 899-908

[14] Ternik, P.; Marn, J.; Žunič, Z. (2006). Non-Newtonian fluid flow through a planar symmetric expansion: Shear-thickening fluids, Journal of Non-Newtonian Fluid Mechanics, Vol. 135, 136148 
[15] Marn, J.; Ternik, P. (2006). Laminar flow of a shear-thickening fluid in a $90^{\circ}$ pipe bend, Fluid Dynamics Research, Vol. 38, 295-312

[16] Marn, J.; Ternik, P. (2003). Use of Quadratic model for modelling of fly-ash water mixture, Applied Rheology, Vol. 13, 286-296

[17] Brodkey, R. S.; Hershey, H. C. (1988). Transport Phenomena, McGraw-Hill, USA

[18] Bird, R. B.; Stewart, W. E.; Lightfoot, E. N. (1960). Transport Phenomena, John Wiley \& Sons, Inc., USA

[19] Arora, D. Computational Fluid Dynamics of Blood Flow: Chein's Experimental Data, from http://www.mems.rice.edu/ dhruv/, accessed on 20.06.2007

[20] Chapra, S. C.; Canale, R. P. (1998). Numerical Methods for Engineers, McGraw-Hill, USA

[21] Leonard, B. P. (1979). A stable and accurate convective modelling procedure based on Quadratic upstream interpolation, Computer Methods in Applied Mechanics and Engineering, Vol. 19, 5998

[22] Van Doormal, J. P.; Raithby, G. D. (1984). Enhancements of the SIMPLE algorithm for predicting incompressible fluid flows, Numerical Heat Transfer, Vol. 7, 147-163 